\title{
Assistência de enfermagem frente ao planejamento familiar na Atenção Primária à
}

\section{Saúde}

Nursing care in the face of family planning in Primary Health Care

La atención de enfermería ante la planificación familiar en la Atención Primaria de Salud

Anamerinda de Oliveira Diaz

ORCID: https://orcid.org/0000-0002-9603-8577 Universidade Estadual do Ceará, Brasil

E-mail: merindaodiaz@gmail.com

Fábio da Silva Rocha

ORCID: https://orcid.org/0000-0001-8552-1670 Faculdade do Belo Jardim, Brasil

E-mail: fabiodasilvarocha310@gmail.com

Laíssa Almeida Custódio da Silva ORCID: https://orcid.org/0000-0003-3115-9375 Centro Universitário Fametro, Brasil E-mail: laissa020@gmail.com

Sabina Dias Rangel

ORCID: https://orcid.org/0000-0001-7299-5543

Universidade Santo Amaro, Brasil

E-mail: sabina.rangel2020@gmail.com

Ana Vitória Lima Marcos

ORCID: https://orcid.org/0000-0002-6896-6624

Centro Universitário Maurício de Nassau, Brasil

E-mail: vitorialima2501@gmail.com

Karina Correia Marques

ORCID: https://orcid.org/0000-0003-3427-1186

Centro Universitário Maurício de Nassau, Brasil

E-mail: karinacorreia2609@gmail.com

Gustavo Nunes de Mesquita

ORCID: https://orcid.org/0000-0002-8643-6029

Centro Universitário de Barra Mansa, Brasil

E-mail: gustavomesquita113@gmail.com

Luiz Fernando de Almeida

ORCID: https://orcid.org/0000-0003-2785-587X

Universidade Federal de São João del-Rei, Brasil

E-mail: luizfernando2204@yahoo.com.br

Vinícius Eugênio da Silva

ORCID: https://orcid.org/0000-0002-7573-7539 Pontifícia Universidade Católica de Minas Gerais, Brasil

E-mail: viniciusenfer@hotmail.com

Wesley Romário Dias Martins

ORCID: https://orcid.org/0000-0002-9270-056X

Faculdade Orígenes Lessa, Brasil

E-mail: wesleyrdm_enf@outlook.com.br 


\begin{abstract}
Resumo
Este estudo tem como objetivo descrever quais são os métodos contraceptivos compreendidos no contexto do planejamento familiar, e compreender a assistência do profissional de enfermagem na Atenção Primária à Saúde. Trata-se de um estudo descritivo, do tipo revisão integrativa de literatura, de caráter qualitativo, realizado por meio da busca de artigos indexados na Biblioteca Virtual em Saúde (BVS), com o auxílio das seguintes bases de dados: Scientific Electronic Library Online (SCIELO), National Center for Biotechnology Information (PUBMED), Literatura Latino-Americana e do Caribe em Ciências da Saúde (LILACS), Base de Dados de Enfermagem (BDENF), e Periódicos Eletrônicos em Psicologia (PEPSIC). Os critérios de inclusão compreendem pesquisas de revisão da literatura disponíveis nos bancos de dados descritos, compreendidos entre os anos de 2010 a 2020. Os critérios de exclusão, foram artigos duplicados, incompletos, resumos, resenhas, relato de experiência, artigos publicados em anais de eventos e indisponíveis na íntegra. Para o levantamento dos dados foram analisados 17 artigos. Os resultados do estudo evidencia que, os principais métodos de planejamento familiar ofertados na Atenção Primária à Saúde são: pílula, minipílula, injetável hormonal, preservativo, laqueadura tubária e vasectomia. O enfermeiro tem como atribuição orientar a população quanto ao modo de uso, eficácia, efeitos colaterais, e implicações para vida sexual. Concluímos que, o planejamento familiar está associado à educação em saúde como um instrumento para prevenção de uma gravidez indesejada, o enfermeiro atua como mediador entre o serviço e a população, buscando melhores estratégias para garantia dos direitos em saúde sexual e reprodutiva.
\end{abstract}

Palavras-chave: Promoção da saúde; Estratégia Saúde da Família; Planejamento familiar.

\begin{abstract}
This study aims to describe which contraceptive methods are understood in the context of family planning, and to understand the nursing professional's assistance in Primary Health Care. This is a descriptive, qualitative, integrative literature review study, carried out by searching for articles indexed in the Virtual Health Library (VHL), with the help of the following databases: Scientific Electronic Library Online (SCIELO), National Center for Biotechnology Information (PUBMED), Latin American Literature and the Caribbean in Health Sciences (LILACS), Nursing Database (BDENF), and Electronic Journals in Psychology (PEPSIC). The inclusion criteria include literature review research available in the databases described, between the years 2010 to 2020. The exclusion criteria were duplicate, incomplete articles, abstracts, reviews, experience reports, articles published in annals of events and unavailable in full. To collect the data, 17 articles were analyzed. The results of the study show that the main methods of family planning offered in Primary Health Care are: pill, mini-pill, hormonal injectable, condom, tubal ligation and vasectomy. The nurse has the task of guiding the population as to the way of use, effectiveness, side effects, and implications for sexual life. We conclude that family planning is associated with health education as an instrument to prevent unwanted pregnancies, the nurse acts as a mediator between the service and the population, seeking better strategies to guarantee sexual and reproductive health rights.
\end{abstract}

Keywords: Health promotion; Family Health Strategy; Family planning.

\title{
Resumen
}

Este estudio tiene como objetivo describir qué métodos anticonceptivos se entienden en el contexto de la planificación familiar, y comprender la asistencia del profesional de enfermería en la Atención Primaria de Salud. Se trata de un estudio de revisión bibliográfica descriptiva, cualitativa e integradora. , realizado mediante la búsqueda de artículos indexados en la Biblioteca Virtual en Salud (BVS), con la ayuda de las siguientes bases de datos: Biblioteca Científica Electrónica en Línea (SCIELO), Centro Nacional de Información Biotecnológica (PUBMED), Literatura Latinoamericana y el Caribbean in Health Sciences (LILACS), Base de datos de enfermería (BDENF) y Revistas electrónicas de psicología (PEPSIC). Los criterios de inclusión incluyen investigación de revisión de la literatura disponible en las bases de datos descritas, entre los años 2010 a 2020. Los criterios de exclusión fueron artículos duplicados, incompletos, resúmenes, revisiones, informes de experiencias, artículos publicados en anales de eventos y no disponible en su totalidad. Para la recogida de datos se analizaron 17 artículos. Los resultados del estudio muestran que los principales métodos de planificación familiar que se ofrecen en Atención Primaria de Salud son: píldora, minipíldora, inyectable hormonal, condón, ligadura de trompas y vasectomía. La enfermera tiene la tarea de orientar a la población en cuanto a la forma de uso, efectividad, efectos secundarios e implicaciones para la vida sexual. Concluimos que la planificación familiar se asocia a la educación en salud como instrumento para prevenir embarazos no deseados, la enfermera actúa como mediadora entre el servicio y la población, buscando mejores estrategias para garantizar los derechos de salud sexual y reproductiva.

Palabras clave: Promoción de la salud; Estrategia de Salud Familiar; Planificación familiar.

\section{Introdução}

A Atenção Primária a Saúde (APS) apresenta um conjunto de ações para a população, desenvolvidas por uma equipe multiprofissional. Ações de promoção, prevenção, proteção, diagnóstico, tratamento e diminuição de danos, compreende as práticas assistenciais voltadas para o indivíduo, família e comunidade (Brasil, 2017). 
As atividades educativas em saúde é considerada uma das ações prioritárias da Atenção Primária à Saúde, sendo assim, entende-se por educação em saúde, um conjunto de ações de saúde que possibilita maior autonomia e conhecimento da população quanto aos cuidados em saúde, tendo em vista a humanização e a assistência integral à saúde (Nunes et al., 2013).

O Sistema Único de Saúde (SUS), visa atender todas as formas de famílias existentes, seja tradicional ou moderna, objetivando um serviço integral e igualitário para toda população (Brasil, 2013). No século XX, o planejamento familiar ganhou grande visibilidade e um importante espaço nas Políticas Públicas de Saúde por estar diretamente relacionado com a decisão de ter filhos, ou não (Bhering, 2016).

Planejar e orientar quanto a escolha do método anticoncepcional é primordial na promoção de saúde, sendo atribuição dos profissionais de saúde fazer o acompanhamento e viabilizar informações sobre as técnicas e práticas quando necessário (Ferreira, Costa \& Melo, 2014).

Segundo Ferreira, Costa e Melo (2014), há uma articulação entre as políticas governamentais e o planejamento familiar, após movimentos sociais que redemocratizou o perfil de controle de natalidade para um direito social. A primeira razão foi denominada pelo controle de natalidade, representada pela crise econômica da época, causando, também o conceito de família ideal e a perspectiva para a diminuição do índice de abortamento (Bhering, 2016).

De acordo com os estudos de Meirelles, Negreiros e Maia (2014) o planejamento familiar surge como uma importante proposta a fim de estimular a população quanto ao uso de métodos contraceptivos, recomendado por profissionais de saúde. Entre os profissionais relacionados a este processo, o enfermeiro é o mais indicado, devido suas atribuições prioritárias de estratégias e ações que promovem a saúde e a qualidade de vida do indivíduo.

De acordo com Maranhão, Gomes e Barros (2016) ao longo dos anos, homens e mulheres conquistaram sua independência quanto a formação familiar, ter ou não ter filhos. Portanto, a maioria dos casais não possuem conhecimento acerca do planejamento familiar, resultando em gestações não planejadas, seguido de insatisfações pessoais, conflitos socioeconômicos e a ocorrência de abortos.

De acordo com Brasil (2004), grande parte dos serviços prestados à população feminina são voltados para prevenção ou tratamento de enfermidades, planejamento reprodutivo, e assistência materna.

A população feminina representa uma maioria na sociedade brasileira, e em número são as que mais usufruem dos benefícios do Sistema Único de Saúde (SUS), tanto para próprio atendimento, quanto para seus filhos, esposo, familiares e pessoas próximas (Brasil, 2004). Falar sobre direitos reprodutivos e sexuais não é tão simples, pois requer muito domínio e habilidade acerca do tema, visto que, trata - se de um assunto polêmico, historicamente marcado por preconceitos e tabus (Brasil, 2013).

A assistência à saúde da mulher nos Serviços de Saúde, não compreende apenas o ciclo puerperal; a assistência à saúde deve ocorrer de modo integral, incluindo a prevenção da gravidez indesejada (Anjos, 2013).

O planejamento familiar associa - se, ao direito reprodutivo e sexual do indivíduo, considerando os aspectos morais e culturais, além de evitar a eventualidade de uma possível gravidez não planejada. Sendo assim, quais são os principais métodos contraceptivos orientados por profissionais da Atenção Primaria à Saúde?

Dessa forma, a importância desse estudo se justifica devido à grande relevância temática visando aprimorar a contribuição para uma assistência qualificada na saúde da mulher, evidenciando os métodos contraceptivos ofertados nos Serviços de Saúde.

Sendo assim, este estudo tem como objetivo descrever quais são os métodos contraceptivos compreendidos no contexto do planejamento familiar, e compreender a assistência do profissional de enfermagem na Atenção Primária à Saúde. 


\section{Metodologia}

Trata-se de uma revisão integrativa de literatura, com abordagem qualitativa. A revisão integrativa da literatura proporciona a identificação, síntese e a execução de uma investigação ampla da literatura acerca de uma temática especifica (Silva et al., 2020). Este método de pesquisa possibilita a composição de múltiplos estudos publicados e proporciona conclusões gerais a respeito de uma particular área de estudo (Polit \& Beck, 2006).

Para Vieira e Zouain (2005) a pesquisa qualitativa concede uma importância fundamental as declarações sociais dos envolvidos, aos discursos e aos significados disseminados por eles. Ou seja, esse tipo de pesquisa estima pela descrição detalhada dos fenômenos e dos elementos envolvidos.

O levantamento dos dados foram realizados entre os meses de janeiro a setembro de 2020, por meio da busca de artigos indexados na Biblioteca Virtual em Saúde (BVS), e Scientific Electronic Library Online (SciELO), com o auxílio das seguintes bases de dados: PubMed (National Center for Biotechnology Information), Lilacs (Literatura Latino-Americana e do Caribe em Ciências da Saúde), Base de Dados de Enfermagem (BDENF), PEPSIC (Periódicos Eletrônicos em Psicologia). Foram localizados os seguintes Descritores em Ciências da Saúde: Promoção da Saúde; Estratégia Saúde da Família; Planejamento Familiar.

Os critérios de inclusão para a seleção dos conteúdos foram, artigos completos na linguagem portuguesa, inglesa e espanhola. Publicados na íntegra de acordo com a temática referente à revisão integrativa, documentos, regulamentações, normativas de entidades de saúde acerca do tema, artigos, capítulos de livros, teses, e dissertações publicados nos referidos bancos de dados compreendendo os anos de 2010 a 2020. Os critérios de exclusão, foram artigos duplicados, incompletos, resumos, resenhas, debates, relato de experiência, artigos publicados em anais de eventos e indisponíveis na íntegra.

\section{Resultados e Discussão}

Com base na revisão de literatura e análise dos estudos indexados nas bases de dados eletrônicas, acerca da temática proposta, foram encontrados 109 estudos científicos, sendo que, apenas 42 estudos foram selecionados, 27 atenderam aos critérios de inclusão previamente estabelecidos, destes, 10 foram excluídos mediante os critérios de exclusão. Restando 17 artigos para composição e análise do estudo. 
Figura 1. Fluxograma de identificação e seleção dos artigos. 2020.

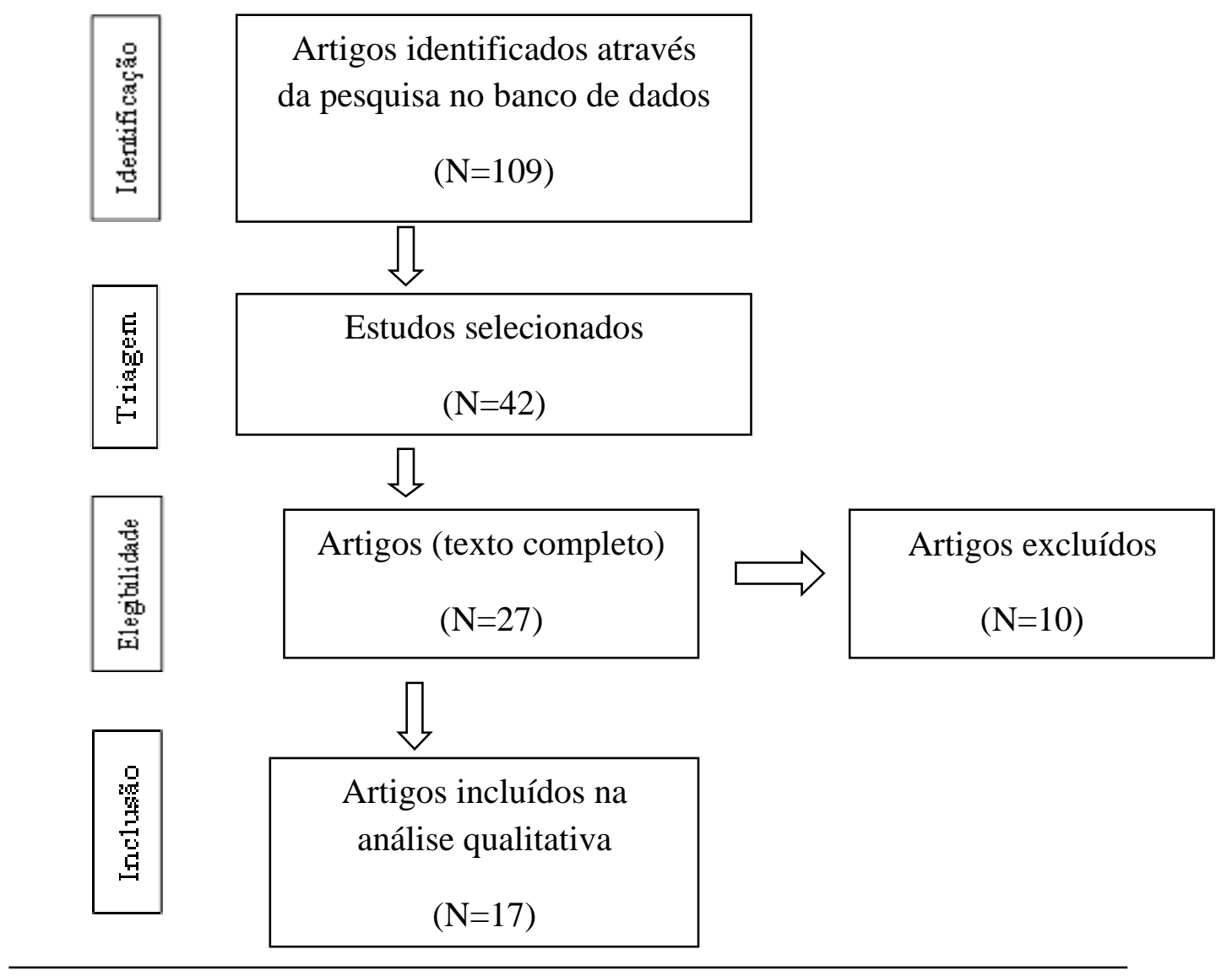

Fonte: Autores (2020).

Santos e Freitas (2011), abordaram que o planejamento familiar envolve alguns marcos importantes para a sociedade, incluindo diversas áreas, especialmente a política, a economia e a psicossocial.

Os estudos de Santos e Freitas (2011) afirmam que, a assistência destinada ao serviço de planejamento familiar não se limita apenas em métodos contraceptivos, ou seja, compreende também a troca de informações, acolhimento humanizado, e orientações para mulheres/casais que procuram o programa. Portanto, é fundamental que os indivíduos memorizem as orientações prestadas pelos profissionais de saúde, a fim de utilizar a técnica correta do método contraceptivo.

Costa et al. (2013) apresenta em seus estudos, a grande variedade de métodos contraceptivos disponíveis nos Serviços de Saúde, compreendendo métodos hormonais (pílulas, adesivos, injeções, implantes cutâneos e anel vaginal); métodos de barreira (preservativo feminino, preservativo masculino, diafragma e espermicidas); dispositivo intrauterino (DIU) de cobre ou com hormônios; contracepção de emergência, divulgada popularmente como pílula do dia seguinte; e métodos definitivos (vasectomia e ligadura de trompas).

O enfermeiro tem como atribuição orientar a população quanto ao modo de uso, eficácia, efeitos colaterais, e implicações para vida sexual (Pierre \& Clapis, 2010).

Os métodos contraceptivos menos oferecidos nos serviços de saúde, são: pílula do dia seguinte, diafragma, e preservativo feminino. Já o aleitamento materno exclusivo, coito interrompido, tabelinha e muco cervical são os métodos contraceptivos menos orientados por profissionais de Saúde (Pierre \& Clapis, 2010). 
De acordo com Brasil (2013), a laqueadura de trompas, pílula e os métodos de livre escolha compreendem os métodos de anticoncepção desde o princípio, sendo considerada uma das ações da Política de Assistência Integral à Saúde da Mulher, preconizada pelo Ministério da Saúde desde o ano de 1984.

Ainda segundo Brasil (2013), a demanda pela laqueadura tubária, esterilização definitiva dentro das alternativas dos métodos contraceptivos, compreendem a maior procura pelo planejamento familiar. Portanto, a execução cirúrgica é criteriosa, compreendendo a idade pertinente, via de parto e quantidade de filhos, além de todo apoio psicológico envolvido no programa.

A lei de número 9.263/96 considera que, a laqueadura só e concedida para mulheres acima de 25 anos com 2 filhos vivos, em aconselhamentos psicológicos decorrente das práticas de métodos definitivos, compreendendo a assistência ao planejamento familiar que considera a oferta de métodos e técnicas para a concepção, anticoncepção, orientações, acompanhamento e acolhimento humanizado em um contexto de livre escolha (Brasil, 2013).

Ferreira, Costa e Melo (2014) dissertam que, o Planejamento Familiar deve ser tratado dentro do contexto dos direitos reprodutivos da mulher. Compreendendo o direito de ter filhos como principal objetivo para garantir as mulheres a escolha de quais métodos contraceptivos usar para evitá-los.

Ferreira, Costa e Melo (2014) apresentam que, ainda há uma grande carência na troca de informações e manuseio dos métodos de contracepção. Assim, se faz necessário uma assistência qualificada pelos profissionais de saúde a fim de atender todas as necessidades das mulheres.

Anjos (2013) considera que, a assistência para as mulheres nos Serviços de Saúde não se enquadra apenas no ciclo puerperal, o cuidado necessita ser de forma integral, incluindo a prevenção da gestação indesejada. O planejamento familiar, amparado pela lei $\mathrm{n}^{\circ}$ 9.263, de 1996, envolve todos aqueles que querem ter uma família ou não, atribuindo ações específicas a esses indivíduos (Brasil, 2013).

Bhering (2016) contextualiza que existe uma associação entre as políticas tradicionais e as políticas antinatalistas, considerando que, são poucos os programas governamentais relacionados ao planejamento familiar.

Além das dificuldades apresentadas, correlação às leis, há muitos avanços relacionados à legislação nacional e internacional sobre os Direitos Humanos e aos assuntos ligados à saúde reprodutiva e sexual do casal (Brasil, 2013).

Assim, é importante ampliar essas abordagens, sendo que a anticoncepção faz parte das ações de planejamento familiar e depende de condições socioeconômicas, culturais, educativas da família, sendo assim, a escolha do método precisa ser mútua entre o casal (Brasil, 2013).

É necessário agir de forma universal para adquirir um amplo envolvimento de homens e mulheres, baseando-se, no respeito e nas responsabilidades que cabem a cada um dos envolvidos. Aos homens, tanto na paternidade e nas prevenções de IST (Infecções Sexualmente Transmissíveis), como também no planejamento familiar, atribuindo aos princípios do SUS (Brasil, 2013).

Costa et al. (2013) consideram que, é possível determinar a quantidade de filhos que o casal pretende ter, sendo que a anticoncepção depende do casal, seguindo a ideologia do liberalismo, que somente o indivíduo pode progredir por ele mesmo.

Para Morais et al. (2014) as mulheres são as que mais procuram os Serviços de Saúde para implementação de métodos contraceptivos. Assim, a responsabilidade das mulheres passa a ser maior do que a responsabilidade dos homens, em decorrência da quantidade existente de métodos contraceptivos que são destinados às mulheres, pois as mulheres estão mais envolvidas aos cuidados e prevenção.

Referente aos direitos igualitários, o Ministério da Saúde afirma que, o poder de exercer a sexualidade e a reprodução deve ser livre de discriminação, imposição e violência. Concedendo a mulher, o direito ao acesso de informações e métodos para ter ou não filhos (Brasil, 2013). 
Costa et al. (2013) comentou sobre o amplo desafio enfrentado durante o desenvolvimento do planejamento familiar e o controle da natalidade. Assim, as informações fornecidas à população, eram consideradas ineficaz pelos Serviços de Saúde.

Moura e Gomes (2014) relatam sobre a baixa adesão da população aos serviços de planejamento familiar, consequentemente, fator resultante da carência de informações profissionais quanto ao uso correto dos métodos contraceptivos.

Alguma das ações realizadas pelas equipes multiprofissionais, como: terapias de grupo, trabalho psicológico, acolhimento, explicação dos métodos disponíveis, e até mesmo gestos simples como escutar o paciente, são decisivos para auxiliar neste processo de planejamento familiar (Lacerda, et al., 2020).

\section{Considerações Finais}

O presente estudo atendeu ao objetivo proposto evidenciando os principais métodos contraceptivos compreendidos no contexto do planejamento familiar, e a contribuição dos profissionais de saúde.

Evidencia - se, que o planejamento familiar está associado à educação em saúde como um instrumento para prevenção de uma gravidez indesejada, o enfermeiro atua como mediador entre o serviço e a população, buscando melhores estratégias para garantia dos direitos em saúde sexual e reprodutiva.

Assim, o processo educativo realizado pelo Enfermeiro é essencial à implementação efetiva do planejamento familiar, apresentando propostas para intensificar o real objetivo do programa compreendendo o desejo de ter filhos ou não, sendo uma das prioridades da Atenção Primária à Saúde.

Existem métodos preventivos destinados tanto as mulheres, quanto aos homens. Sendo assim, é inadmissível que a mulher carregue sozinha a culpa pela gestação não planejada, mesmo que haja uma resistência masculina em procurar os Serviços de Saúde, é de extrema relevância que ambas as partes se responsabilizem pelo filho não planejado.

\section{Referências}

Anjos, K. F., Santos, V. C., Souzas, R., \& Eugênio, B. G. (2013). Aborto e saúde pública no Brasil: reflexões sob a perspectiva dos direitos humanos. Saúde em Debate, 37(98), 504-515.

Bhering, M. J. (2016). História do Planejamento Familiar. História, Ciência e Saúde, 23(1), 224-48.

Brasil. (2013). Ministério da saúde. Saúde sexual e reprodutiva. Caderno de atenção básica. Secretaria de Atenção à Saúde. Brasília (DF):ministério d saúde, 2013. Recuperado em 14 de setembro de 2018, http://189.28.128.100/dab/docs/portaldab/publicacoes/cab13.pdf

Brasil. (2017). Ministério da Saúde. Portaria n².436 DE 21 de Setembro de 2017. Aprova a nova Política Nacional de Atenção Básica. 2017.

Costa, A., Rosado, L., Florêncio, A., \& Xavier, E. (2013). História do Planejamento familiar e sua relação com os métodos contraceptivos. Revista Baiana de Saúde Pública, 37(1),74-86.

Ferreira, R. V., Costa, M. R., \& Melo, C. S. (2014). Planejamento Familiar: gênero e significados. Textos \& contextos, 13(2),387-97.

Lacerda, C. S., Balbino, C. M., Sá, S. P. C., Silvino, Z. R., Silva Júnior, P. F., Gomes, E. N. F., \& Joaquim, F. L. (2020). Confrontation of women with breast cancer. Research, Society and Development, 9(7), 1-18.

Maranhão, T. A., Gomes, K. R. O., \& Barros, I. C. (2016). Fatores atribuídos ao aborto entre jovens com experiência obstétrica. Revista Brasileira de Epidemiologia, 19 (3), 494-508.

Morais, A. C. B., Ferreira, A. G., Almeida, K. L., \& Quirino, G. S. (2014). Participação masculina no planejamento familiar e seus fatores intervenientes. Revista de Enfermagem da UFSM, 4(3), 498-508.

Moura, L. N. B., \& Gomes, K. R. O. (2014). Planejamento familiar: uso dos serviços de saúde por jovens com experiência de gravidez. Ciência \& Saúde Coletiva, 19(3), 853-863.

Polit, D. F.; \& Beck, C. T. (2006). Using research in evidence-based nursing practice. In: Polit, D. F. Beck, C. T. Editors. Essentials of nursing research. Methods, appraisal and utilization. Philadelphia (USA): Lippincott Williams \& Wilkins. p. 457-94. 
Research, Society and Development, v. 10, n. 1, e45710110506, 2021

(CC BY 4.0) | ISSN 2525-3409 | DOI: http://dx.doi.org/10.33448/rsd-v10i1.10506

Santos, J. C., \& Freitas, P. M. (2011). Planejamento familiar na perspectiva do desenvolvimento. Ciência \& Saúde Coletiva, 16 (3), $1813-20$.

Silva, C. C., Savian, C. M., Prevedello, B. P., Zamberlan, C., Dalpian, D. M., \& Santos, B. Z. dos. (2020). Access and use of dental services by pregnant women: An integrative literature review. Ciencia e Saude Coletiva, 25 (3), 827- 835. 